\title{
Serum Chromium and Gestational Diabetes
}

\author{
Scott E. Woods, MD, MPH, MEd, Vivian Ghodsi, RN, Amy Engel, MA, Jeff Miller, BS, \\ and Shama James, $M D$
}

Objective: To prospectively assess if chromium levels are different in gestational diabetics than in nondiabetic pregnant women. Therapeutic options could exist if levels are different.

Methods: We conducted a 2-year prospective, gestational cohort study. The cohort included newly diagnosed pregnant patients. Two blood samples were taken, the first at consent, the second at the time of diabetes screening.

Results: Five hundred eighty patients entered the cohort. One hundred fifty-five were lost to follow up and 425 patients completed the study (396 nondiabetics and 29 diabetics). The patients with gestational diabetes were significantly older, with a family history of gestational diabetes, and were significantly more likely to have been a gestational diabetic in a previous pregnancy $(P<05)$. There was no significant difference between the 2 groups for chromium levels both at enrollment $(0.15 \pm 0.06 \mathrm{ng} / \mathrm{mL}$ vs $0.14 \pm 0.03 \mathrm{ng} / \mathrm{mL} ; P=.28)$ and during the second trimester $(0.14 \pm 0.06 \mathrm{ng} / \mathrm{mL}$ vs $0.14 \pm 0.04$ $\mathrm{ng} / \mathrm{mL} ; \boldsymbol{P}=.82)$.

Conclusion: Serum chromium levels do not exhibit any significant variation between gestational diabetic women and nondiabetic women when assessed prospectively. (J Am Board Fam Med 2008;21: 153-157.)

Chromium is a mineral found in many foods, including liver, cheese, meats, fish, fruits, vegetables, and whole grains. It is believed to play a role in glucose homeostasis by acting as a cofactor for insulin, and chromium deficiency has been observed to have a significant effect on glucose intolerance. ${ }^{1}$ Most multivitamins provide well above the recommended daily requirement of chromium (20-25 mcg for adult women and 30-35 mcg for adult men). Some prenatal vitamins contain chromium but others do not.

Nutrient requirements increase during normal pregnancy, in part because of increased demand but also because of increased excretion. Early research by Davidson ${ }^{2}$ showed that plasma chromium con-

This article was externally peer reviewed.

Submitted 20 June 2007; revised 3 October 2007; accepted 5 October 2007.

From Bethesda Family Medicine Residency Program (SEW, SJ); the E. Kenneth Hatton, MD, Institute for Research and Education (VG, AE, JM), Cincinnati, OH.

Funding: Financial support provided by the Bethesda Foundation, the Good Samaritan and the Good Samaritan Hospital Foundation.

Conflict of interest: none declared.

Corresponding author: Scott E. Woods, MD, MPH, MEd, Associate Program Director, Bethesda Family Medicine Residency Program, 4411 Montgomery Road, Suite 200, Cincinnati, OH 45212 (E-mail: Liverdoctor@yahoo.com). centrations were substantially lower than normal in pregnancy and that this may represent normal physiology as well as depletion of maternal stores. A study examining chromium excretion during pregnancy found that persistently elevated excretion in otherwise normal pregnancies seemed to be associated with a significant reduction in insulin sensitivity. ${ }^{3}$ In turn, it was speculated that enhanced chromium excretion had the potential to put a mother at increased risk of developing gestational or postpartum diabetes.

However, the influence of chromium on gestational diabetes continues to be a point of contention. This is partly because of the lack of consensus on what method is best for measuring chromium levels. One study looked at hair chromium concentration in relation to gestational diabetes. ${ }^{4}$ It demonstrated that hair chromium concentration was significantly higher in patients with gestational diabetes and that the disease was not associated with low chromium status as researchers believed. The study, instead, suggested that the underlying cause of glucose intolerance was the impaired use of chromium. $^{4}$ A separate prospective study used plasma chromium to assess chromium levels. ${ }^{5}$ It concluded that plasma chromium during pregnancy did not correlate with glucose intolerance, 
insulin resistance, or serum lipid levels. However, it also noted that plasma chromium may not reflect tissue stores of chromium and that a method for assessing body chromium stores was required for further studies. ${ }^{5}$ The best test for assessing chromium is unknown and it may be different for acute versus chronic disease states. We choose to evaluate serum using a new technique because we were evaluating a subacute disease and the new device is more accurate than previous testing methods.

Our goal was to prospectively quantify the serum chromium levels in pregnant women and assess if there was any correlation between chromium levels and gestational diabetes. This will be the first study to analyze serum during the first and second trimester of pregnancy for comparison. We also used a new, highly accurate device, the argon gas Inductively Coupled Plasma Mass Spectrometer (ICP-MS; PerkinElmer Life and Analytical Sciences, Inc., Waltham, MA), for the analysis. We did not have a hypothesis for this project.

\section{Methods}

We conducted a 2.5-year prospective gestational cohort study. The cohort included newly diagnosed pregnant patients presenting to the routine (not high risk) obstetrics and gynecology clinic at Good Samaritan Hospital, Cincinnati, Ohio, from February 2004 to July 2006. We excluded patients who had already been diagnosed with gestational diabetes for this pregnancy, type 1 diabetics, type 2 diabetics, and patients presenting outside of the first trimester at their first visit. A research nurse dedicated to this study interviewed all newly diagnosed pregnant patients. After consent was given, a single tube of blood was taken from each patient in a special trace-free metal vacutainer tube. We rapidly transported all samples to the Hatton Research Lab (located in the same hospital) for sub-zero storage. At the initial visit, data were collected about age, race, smoking status, parity, family history of gestational diabetes, previous history of gestational diabetes, medications (including supplements), and education level. A second blood sample was taken at the time of screening for gestational diabetes (typically around 25-28 weeks), with the same special trace-free metal vacutainer tube. We recorded the gestational diabetes status for the current pregnancy after this visit as well as the treatment for gestational diabetics in the cohort. We used the American College of Obstetricians and Gynecologists criteria for defining patients as being a gestational diabetic. Participants exited the cohort after the second blood sample was drawn. Institutional Review Board approval was obtained before data collection.

All serum samples were introduced to the argon gas ICP-MS instrument. The ICP-MS is the newest method for ultratrace metal analysis. This process offers several advantages over more traditional graphite furnace atomic absorption spectrometry. Samples and standards are introduced to the ICP-MS instrument via a peristaltic pump at the rate of $1 \mathrm{~mL} / \mathrm{min}$. The sample is then subjected to a $10,000^{\circ}$ plasma torch. The extreme heat of this torch completely breaks apart molecules present in the sample matrix. The extreme heat also forces an electron off of the element, creating a positively charged elemental atom. The positron is then subjected to polarity changes within a quadrupole magnet at high vacuum pressures. This polarity change happens several thousand times per second. As a result, the element is slowed down in the magnet by its atomic mass. Low-mass elements travel through the quadrupole at a greater speed than heavier atoms, such as lead or uranium. A detector then counts the individual positively charged elemental atoms according to their atomic weight. Finally the instrument sends this information to a high-speed computer that processes the data. The ICP-MS is capable of performing multiple analyses from a single sample at parts per trillion levels or lower. For the element chromium, the ICP-MS is accurate to 1.0 parts per trillion.

Using a 2 -tailed $\alpha$ of .05 and a $\beta$ of .10, our study would require approximately 500 patients to be able to find a $17 \%$ difference in serum chromium levels statistically significant with $90 \%$ power. This also assumes that gestational diabetes will occur in approximately $5 \%$ of the study population.

\section{Results}

Twenty-seven patients refused to be in the study. Five hundred eighty patients initially consented and entered the cohort. One hundred fifty-five were lost to follow up (miscarriages, change of obstetrical care, moved, or did not return), leaving 425 patients to complete the cohort study (396 nondiabetics; 29 gestational diabetics [15 
Table 1. Comparison of Nondiabetic Women with Those with Gestational Diabetes

\begin{tabular}{|c|c|c|c|}
\hline Variable & $\begin{array}{l}\text { Nondiabetics } \\
\quad(\mathrm{n}=396)\end{array}$ & $\begin{array}{l}\text { Gestational Diabetics } \\
\qquad(\mathrm{n}=29)\end{array}$ & $P$ \\
\hline Age (years) & $24.1 \pm 4.9$ & $27.7 \pm 5.2$ & $<.01$ \\
\hline \multicolumn{4}{|l|}{ Gestation (weeks) } \\
\hline First analysis & $8.5 \pm 2.3$ & $8.4 \pm 2.3$ & 0.79 \\
\hline Second analysis & $26.8 \pm 2.6$ & $25.0 \pm 4.5$ & .03 \\
\hline Parity (n [\%]) & & & 0.06 \\
\hline 0 & $142(35.9)$ & $12(41.4)$ & \\
\hline 1 & $115(29.0)$ & $4(13.8)$ & \\
\hline 2 & $90(22.7)$ & $5(17.2)$ & \\
\hline 3 or more & $49(12.4)$ & $8(27.6)$ & \\
\hline Race (n [\%]) & & & 0.87 \\
\hline Caucasian & $219(55.6)$ & $14(48.3)$ & \\
\hline African-American & $171(43.4)$ & $14(48.3)$ & \\
\hline Other & $4(1.0)$ & $1(3.4)$ & \\
\hline Education (n [\%]) & & & 0.72 \\
\hline$<$ High school & $130(32.8)$ & $8(27.6)$ & \\
\hline High school & $154(38.9)$ & $13(44.8)$ & \\
\hline Some college & $86(21.7)$ & $5(17.2)$ & \\
\hline College graduate & $22(5.6)$ & $3(10.3)$ & \\
\hline Graduate school & $4(1.0)$ & $0(0.0)$ & \\
\hline Smoker (n [\%]) & & & 0.93 \\
\hline Yes & $229(57.8)$ & $17(58.6)$ & \\
\hline No & $167(42.2)$ & $12(41.4)$ & \\
\hline History of gestational diabetes (n [\%]) & & & $<.01$ \\
\hline Yes & $12(3.0)$ & $7(24.0)$ & \\
\hline No & $384(97.0)$ & $22(76.0)$ & \\
\hline Family history/gestational diabetes (n [\%]) & & & .09 \\
\hline Yes & $44(11.3)$ & $6(22.2)$ & \\
\hline No & $346(88.7)$ & $21(77.8)$ & \\
\hline \multicolumn{4}{|l|}{ Chromium level (ng/mL) } \\
\hline Initial (8 weeks' gestation) & $0.15(0.06)$ & $0.14(0.03)$ & .28 \\
\hline Follow-up (26 weeks' gestation) & $0.14(0.06)$ & $0.14(0.04)$ & .82 \\
\hline
\end{tabular}

diet controlled, 14 medication controlled]). Compared with the nondiabetics, the patients with gestational diabetes were significantly older and significantly more likely to have been a gestational diabetic in a previous pregnancy $(P<$ .05 ; Table 1). There was no difference between the 2 groups for race, parity, educational level, smoking habits, or family history of gestational diabetes. The 2 groups were of similar weeks' gestation at enrollment; however, the diabetics were at a younger gestational point at the second blood draw. There was no significant difference between the 2 groups' chromium levels, measurement of which was performed at both enrollment during the first trimester (Table 1) and at the time of the patient's glucose tolerance test.

\section{Discussion}

Our prospective data collected for more than 2 years strongly suggests that serum chromium levels do not exhibit any significant variation between gestational diabetic and nondiabetic women. These results are similar to those found by other researchers. ${ }^{4,5}$ If chromium has any role in gestational diabetes, it may be that it is a possible treatment but not a cause or correlation. It is possible that gestational diabetes is different from type 2 diabetes in regard to chromium. Gestational diabetes is a subacute diagnosis lasting only weeks whereas type 2 diabetes is a chronic condition lasting years to decades. It is possible that long-term chromium levels could play an enhanced role in a chronic condition than in a briefer subacute condition. Recent small 
clinical trials suggest this may be true. Chromium has been found to reduce glucose intolerance and improve lipid levels in elderly diabetic patients ${ }^{6}$; lower glucose levels, insulin resistance, and oxidative stress in obese patients with type 2 diabetes ${ }^{7,8}$; and, in combination with biotin in poorly controlled diabetics, improves glucose management and lipid measurements. ${ }^{9}$ However, a small trial $(n=46)$ of obese, insulin-treated type 2 diabetics, chromium had no significant impact on glucose management after a 6 -month trial. ${ }^{10}$

The research on chromium supplementation for women with gestational diabetes is mixed. A clinical trial by Jovanovic et $\mathrm{al}^{11}$ showed that supplementation with chromium picolinate at $4 \mu \mathrm{g} / \mathrm{kg} / \mathrm{d} /$ body weight lowered HbA1c, glucose, and insulin levels compared with patients' baseline and, therefore, improved glucose intolerance and lowered hyperinsulinemia. Another study by Morris ${ }^{12}$ found that healthy patients given chromium picolinate supplementation for 10 weeks showed significant improvement in their response to endogenous insulin. Nevertheless, the efficacy of the treatment continues to be debatable. A meta-analysis of randomized controlled trials using chromium supplementation by Althuis et $\mathrm{al}^{13}$ concluded that chromium had no effect on glucose or insulin concentrations in nondiabetic patients and that data from diabetic patients was inconclusive. In our study, we asked all women about dietary supplements, including which prenatal vitamin(s), if any, they were taking. Very few women were taking a vitamin with chromium. However, we found the chromium levels in these few women were no different from women who were not taking a supplement with chromium.

It is important to consider the limitations of our project when discussing the results. It could be possible that the patients who refused to be in study were significantly different from those who consented. However, because the number of patients who refused was very small, this is unlikely. The patients in our cohort are exclusively from southwest Ohio and representative of only 2 races. $\mathrm{Pa}$ tients from other geographical areas or other racial backgrounds could be different. Ideally, the second blood draw would have been performed at a similar gestational age in both groups. The diabetic patients were sampled approximately 10 days earlier than were the nondiabetic patients. Although it is difficult to know, we did not believe that the timing difference would significantly impact the results. In addition, there are additional metabolic pathways that effect chromium metabolism, such as iron, which we did not consider in our study. Finally, because of a higher than expected number of participants not returning after the first visit $(27 \% ; 155$ of 580), we did not reach our goal of 500 patients in the cohort. We included as many participants as our grant funds would support. Seventy-five more patients, however, would not have made any statistical difference because the 2 groups were virtually identical at the second blood draw.

\section{Conclusion}

Serum chromium levels do not exhibit any significant variation between gestational diabetic and nondiabetic women when assessed prospectively. Future research should focus on chromium and its role in diabetes, if any, because there is conflicting data in this area. Chromium may have different influences in gestational, type I, and type 2 diabetes. It is also possible that chromium could exhibit different impacts in insulin-dependent and non insulin-dependent patients. Exploring the best test for chromium is also important. Tissue levels may be better for long-term conditions such as type 1 and 2 diabetes. Thus it would be valuable to investigate the best tissue sample for providing accurate assessments of chromium storage in the body.

\section{References}

1. Jovanovic-Peterson L, Peterson C. Vitamin and mineral deficiencies which may predispose to glucose intolerance of pregnancy. J Am Coll Nutr 1996;15: 14-20.

2. Davidson I, Burt R. Physiologic changes in plasma chromium of normal and pregnant women: effect of a glucose load. Am J Obstet Gyn 1973;116:601-8.

3. Morris BW, Samaniego S, Fraser R, MacNeil S. Increased chromium excretion in pregnancy is associated with insulin resistance. J Trace Elem Exper Med 2000;13:389-96.

4. Aharoni A, Tesler B, Paltieli Y, Tal J, Dori Z, Sharf M. Hair chromium content of women with gestational diabetes compared with nondiabetic pregnant women. Am J Clin Nutr 1992;55:104-7.

5. Gunton JE, Hams G, Hitchman R, McElduff A. Serum chromium does not predict glucose tolerance in late pregnancy. Am J Clin Nutr 2001;73:99-104.

6. Rabinovitz H, Friedensohn A, Leibovitz A, Gabay G, Rocas C, Habot B. Effect of chromium supplementation on blood glucose and lipid levels in type 2 
diabetes mellitus elderly patients. Int J Vitam Nutr Res 2004;74:178-82.

7. Vladeva SV, Terzieva DD, Arabadjiiska DT. Effect of chromium on the insulin resistance in patients with type II diabetes mellitus. Folia Med 2005;47: 59-62.

8. Racek J, Trefil L, Rajdl D, Mudrova V, Hunter D, Senft V. Influence of chromium-enriched yeast on blood glucose and insulin variables, blood lipids, and markers of oxidative stress in subjects with type 2 diabetes mellitus. Biol Trace Elem Res 2006;109:215-30.

9. Singer GM, Geohas J. The effect of chromium picolinate and biotin supplementation on glycemic control in poorly controlled patients with type 2 diabetes mellitus: a placebo-controlled, doubleblinded, randomized trial. Diabetes Technol Ther 2006;8:636-43.
10. Kleefstra N, Houweling ST, Jansman FG, et al. Chromium treatment has no effect inpatients with poorly controlled, insulin-treated type 2 diabetes in an obese Western population: a randomized, double-blind, placebo-controlled trial. Diabetes Care 2006;29:521-6.

11. Jovanovic L, Gutierrez M, Peterson C. Chromium supplementation for women with gestational diabetes mellitus. J Trace Elem Exper Med 1999;12: 91-7.

12. Morris B. Chromium action and glucose homeostasis. J Trace Elem Exper Med 1999;12:61-70.

13. Althuis MD, Jordan NE, Ludington EA, and JT Wittes. Glucose and insulin responses to dietary chromium supplements: a meta-analysis. Am J Clin Nutr 2002;76:148-55. 